\title{
Useful Solutions for Plane Wave Diffraction by Dielectric Slabs and Wedges
}

\author{
Gianluca Gennarelli and Giovanni Riccio \\ D.I.E.I.I., University of Salerno, Via Ponte Don Melillo, Salerno, 84084 Fisciano, Italy \\ Correspondence should be addressed to Giovanni Riccio, griccio@unisa.it \\ Received 8 March 2012; Accepted 5 April 2012 \\ Academic Editor: Francesco Soldovieri
}

Copyright (๑) 2012 G. Gennarelli and G. Riccio. This is an open access article distributed under the Creative Commons Attribution License, which permits unrestricted use, distribution, and reproduction in any medium, provided the original work is properly cited.

\begin{abstract}
This work presents an overview of available uniform asymptotic physical optics solutions for evaluating the plane wave diffraction by some canonical geometries of large interest: dielectric slabs and wedges. Such solutions are based on a physical optics approximation of the electric and magnetic equivalent surface currents in the involved scattering integrals. The resulting diffraction coefficients are expressed in terms of the geometrical optics response of the considered structure and the standard transition function of the Uniform Geometrical Theory of Diffraction. Numerical tests and comparisons make evident the effectiveness and reliability of the presented solutions.
\end{abstract}

\section{Introduction}

As well-known, the ability to describe and solve electromagnetic scattering problems is highly valued in many areas such as radio planning, remote sensing for monitoring and surveillance of ground, structures and infrastructures, and through-wall building imaging. Numerical techniques represent a possible answer, but they have an inherent drawback: the computation becomes very intensive (if not unmanageable) at high frequencies, where asymptotic methods based on ray-tracing work more efficiently. In this framework, the Geometrical Theory of Diffraction (GTD) [1] and its uniform version (UTD) [2] have received considerable attention because of their peculiarities. When using GTD or UTD, the first step is to resolve the original problem into smaller and simpler components, each related to a canonical geometry, so that the total solution is a superposition of the contributions from each canonical problem. In other words, such ray-based methods allow one to solve a large number of real scattering problems by using the solutions of a relatively small number of simple problems. In addition, they are easy to apply, provide physical insight into the radiation and scattering mechanisms arising from the various parts of the structure and can be combined with numerically rigorous techniques to obtain hybrid methods.

This paper presents a review of Uniform Asymptotic Physical Optics (UAPO) solutions for diffraction problems concerning some typical canonical structures: dielectric slabs (individually considered or forming junctions) and wedges. Examples of application are relevant to (a) through-wall building imaging [3], where the structure can be decomposed into plates forming junctions and corners with other plates and to (b) radar imaging for ground observation, where the presence of ruptures caused by natural disasters can be represented in the form of a wedge-shaped fracture [4].

The starting point for obtaining a UAPO solution is that of considering the scattering integral and using a $\mathrm{PO}$ approximation of the electric and magnetic surface currents related to the boundary of the object. A further useful approximation and a uniform asymptotic evaluation of the resulting integrals allow one to obtain the diffraction coefficients in the UTD framework. They result to be expressed in terms of the reflection and transmission coefficients of the structure and the standard transition function of UTD. Note that also the heuristic solutions [5-7] have such characteristics, but they 
are not derived by means of a rigorous analytical approach. Moreover, the time domain UAPO diffraction coefficients can be determined according to [8] by taking advantage of the UTD-like formulation of their frequency domain counterparts. In light of the above statements, the UAPO solutions have the same effectiveness and ease of handling of those derived in the UTD context and, in addition, they have the inherent advantage of providing the diffracted field from the knowledge of the geometrical optics (GO) field as the heuristic solutions.

The remainder of this paper is organized as follows. Section 2 is devoted to the plane wave diffraction from the edge of a half plane used to model a truncated thin dielectric slab with finite conductivity. The PO surface currents in the scattering integral are equivalent sources originated by the discontinuities of the tangential GO field components across the layer. The UAPO solution presented in Section 2 can be extended to junctions by taking into account the diffraction contributions of the slabs separately. Of course, this useful feature is due to the linearity of the PO scattering integral. A lossless dielectric wedge with obtuse apex angle and arbitrary permittivity is considered in Section 3. Two separate plane wave diffraction problems relevant to the dielectric region and the surrounding space are tackled and solved by using equivalent PO surface currents lying on the internal and external faces of the wedge. Concluding remarks and future investigations are highlighted in Section 4.

\section{Dielectric Slabs}

The diffraction problem considered in this Section refers to a linearly polarised plane wave impinging on a thin dielectric slab characterised by thickness $d$, relative complex permittivity $\varepsilon_{r}$, and relative permeability $\mu_{r}=1$ and represented by a lossy penetrable half plane surrounded by free space (see Figure 1). The angles $\beta^{\prime}$ and $\phi^{\prime}$ fix the incidence direction: the first is a measure of the skewness with respect to the edge $\left(\beta^{\prime}=90^{\circ}\right.$ corresponds to the normal incidence), and the latter gives the aperture of the edge-fixed plane of incidence with respect to the illuminated face $\left(\phi^{\prime}=0\right.$ corresponds to the grazing incidence). The observation direction is specified by the angles $\beta$ and $\phi$. The $\mathrm{e}^{j \omega t}$ time dependence is assumed and suppressed in the following.

The field scattered at the observation point $P$ can be evaluated by using a PO approximation of the electric $\left(J_{s}^{\mathrm{PO}}\right)$ and magnetic $\left(J_{m s}^{\mathrm{PO}}\right)$ surface currents involved in the wellknown integral:

$$
\underline{E}^{s}=-j k_{0} \iint_{S}\left[(\underline{\underline{I}}-\hat{R} \hat{R}) \zeta_{0} J_{s}^{\mathrm{PO}}+\underline{J}_{m s}^{\mathrm{PO}} \times \hat{R}\right] G\left(\underline{r}, \underline{r}^{\prime}\right) d S
$$

where $\zeta_{0}$ is the free-space impedance, $k_{0}$ is the free-space propagation constant, $\underline{r}=x \hat{x}+y \hat{y}+z \hat{z}=\rho+z \hat{z}$, and $\underline{r}^{\prime}=x^{\prime} \hat{x}+z^{\prime} \hat{z}=\rho^{\prime}+z^{\prime} \hat{z}$ are the position vectors of $P$ and

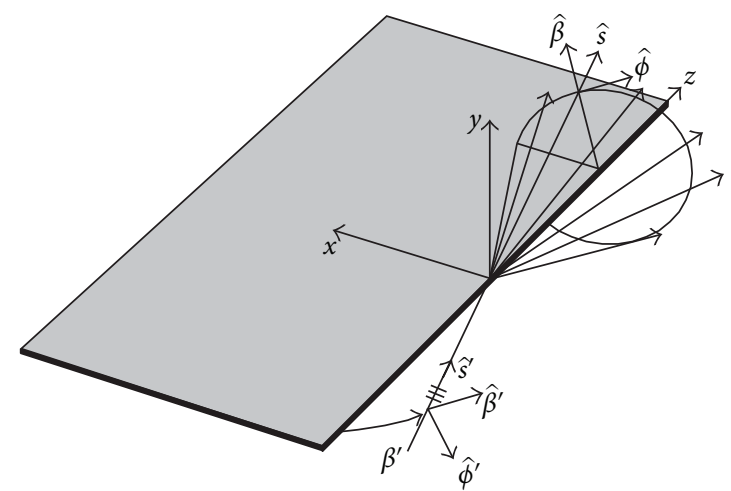

Figure 1: Diffraction by a thin dielectric slab.

the source point, respectively, $\hat{R}$ is the unit vector from the source point to $P, \underline{\underline{I}}$ is the $3 \times 3$ identity matrix, and

$$
\begin{aligned}
G\left(\underline{r}, \underline{r}^{\prime}\right) & =\frac{\mathrm{e}^{-j k_{0}\left|\underline{r}-\underline{r}^{\prime}\right|}}{4 \pi\left|\underline{r}-\underline{\underline{r}^{\prime}}\right|}=\frac{\mathrm{e}^{-j k_{0} \sqrt{\left(x-x^{\prime}\right)^{2}+y^{2}+\left(z-z^{\prime}\right)^{2}}}}{4 \pi \sqrt{\left(x-x^{\prime}\right)^{2}+y^{2}+\left(z-z^{\prime}\right)^{2}}} \\
& =\frac{\mathrm{e}^{-j k_{0} \sqrt{\left|\underline{\rho}-\underline{\rho}^{\prime}\right|^{2}+\left(z-z^{\prime}\right)^{2}}}}{4 \pi \sqrt{\left|\underline{\rho}-\underline{\rho^{\prime}}\right|^{2}+\left(z-z^{\prime}\right)^{2}}}
\end{aligned}
$$

is the three-dimensional Green's function. To evaluate the edge diffraction confined to the Keller's cone $\left(\beta=\beta^{\prime}\right)$, it is possible to approximate $\hat{R}$ by the unit vector $\widehat{s}$ in the diffraction direction, that is, $\hat{R} \cong \hat{s}=\sin \beta^{\prime} \cos \phi \hat{x}+$ $\sin \beta^{\prime} \sin \phi \hat{y}+\cos \beta^{\prime} \hat{z}$. Accordingly, it results that

$$
\begin{aligned}
& \underline{E}^{s}=-\frac{j k_{0}}{4 \pi}\left[(\underline{I}-\widehat{s} \hat{s}) \zeta_{0} \underline{J}_{s}^{*}+\underline{J}_{m s}^{*} \times \hat{s}\right] \\
& \int_{0}^{\infty} \int_{-\infty}^{\infty} \mathrm{e}^{j k_{0}\left(x^{\prime} \sin \beta^{\prime} \cos \phi^{\prime}-z^{\prime} \cos \beta^{\prime}\right)} \frac{\mathrm{e}^{-j k_{0} \sqrt{\left|\underline{\rho}-\underline{\rho}^{\prime}\right|^{2}+\left(z-z^{\prime}\right)^{2}}}}{\sqrt{\mid \underline{\rho}-\underline{\left.\rho^{\prime}\right|^{2}}+\left(z-z^{\prime}\right)^{2}}} d z^{\prime} d x^{\prime} \\
&=\left[(\underline{I}-\hat{s} \hat{s}) \zeta_{0} \underline{J}_{s}^{*}+\underline{J}_{m s}^{*} \times \hat{s}\right] I^{s} .
\end{aligned}
$$

The expressions of the PO surface currents are obtained in terms of the incident electric field $\underline{E}^{i}$ by assuming such currents as equivalent sources originated by the discontinuities of the tangential GO field components across the slab, and then

$$
\begin{aligned}
\zeta_{0} J_{s}^{*} & =\left(1-R_{\perp}-T_{\perp}\right) E_{\perp}^{i} \cos \theta^{i} \hat{e}_{\perp}+\left(1+R_{\|}-T_{\|}\right) E_{\|}^{i}\left(\hat{y} \times \hat{e}_{\perp}\right), \\
\underline{J}_{m s}^{*} & =\left(1-R_{\|}-T_{\|}\right) E_{\|}^{i} \cos \theta^{i} \hat{e}_{\perp}-\left(1+R_{\perp}-T_{\perp}\right) E_{\perp}^{i}\left(\hat{y} \times \hat{e}_{\perp}\right),
\end{aligned}
$$

where $\theta^{i}$ is the standard incidence angle and $\hat{e}_{\perp}$ is the unit vector perpendicular to the plane of incidence. The reflection 


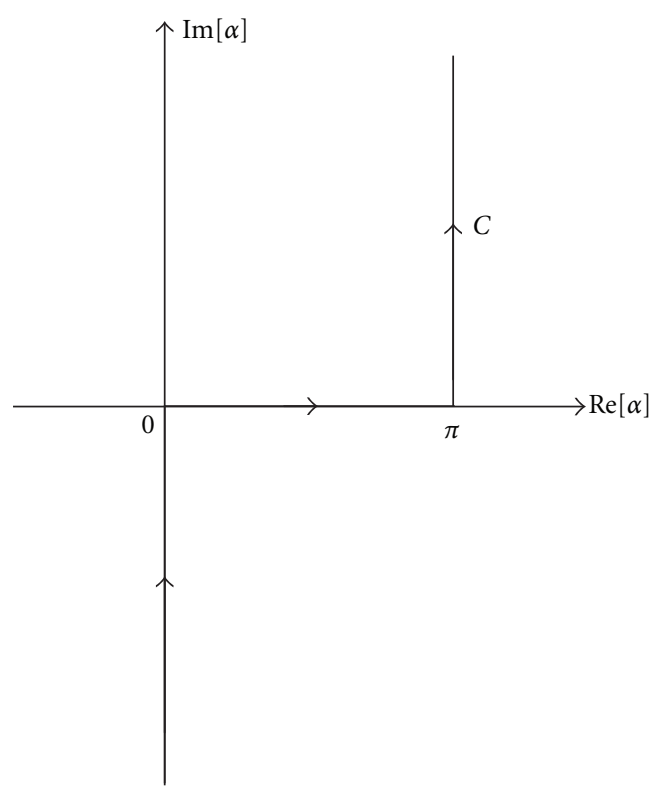

FIgURE 2: Integration path $C$.

$(R)$ and transmission ( $T$ ) coefficients for the parallel ( $\|)$ and perpendicular $(\perp)$ polarisations are given by [9]:

$$
\begin{gathered}
R_{\|, \perp}=\frac{\bar{R}_{\|, \perp}\left[1-\mathrm{e}^{-j 2 \beta_{e q} d / \cos \theta^{t}} \mathrm{e}^{-2 \alpha_{e q} d} \mathrm{e}^{\left.j 2 k_{0} d \sin \theta^{i} \tan \theta^{t}\right]}\right.}{1-\left(\bar{R}_{\|, \perp}\right)^{2} \mathrm{e}^{-j 2 \beta_{e q} d / \cos \theta^{t}} \mathrm{e}^{-2 \alpha_{e q} d} \mathrm{e}^{j 2 k_{0} d \sin \theta^{i} \tan \theta^{t}},} \\
T_{\|, \perp}=\frac{\left[1-\left(\bar{R}_{\|, \perp}\right)^{2}\right] \mathrm{e}^{-j \beta_{e q} d / \cos \theta^{t}} \mathrm{e}^{-\alpha_{e q} d} \mathrm{e}^{j k_{0} d \cos \left(\theta^{i}-\theta^{t}\right) / \cos \theta^{t}}}{1-\left(\bar{R}_{\|, \perp}\right)^{2} \mathrm{e}^{-j 2 \beta_{e q} d / \cos \theta^{t}} \mathrm{e}^{-2 \alpha_{e q} d} \mathrm{e}^{j 2 k_{0} d \sin \theta^{i} \tan \theta^{t}}},
\end{gathered}
$$

in which

$$
\begin{aligned}
\bar{R}_{\|} & =\frac{\varepsilon_{r} \cos \theta^{i}-\sqrt{\varepsilon_{r}-\sin ^{2} \theta^{i}}}{\varepsilon_{r} \cos \theta^{i}+\sqrt{\varepsilon_{r}-\sin ^{2} \theta^{i}}}, \\
\bar{R}_{\perp} & =\frac{\cos \theta^{i}-\sqrt{\varepsilon_{r}-\sin ^{2} \theta^{i}}}{\cos \theta^{i}+\sqrt{\varepsilon_{r}-\sin ^{2} \theta^{i}}} .
\end{aligned}
$$

Formulas (5) and (6) account for the propagation of a nonuniform wave in the layer as considered in [10]. The terms $\beta_{e q}$ and $\alpha_{e q}$ are the equivalent phase and attenuation factors and $\theta^{t}$ is the angle (labelled $\psi_{2}$ in [10]) between the direction of propagation in the layer and the direction normal to the surface (direction of attenuation).

According to (4), (3) can be so rewritten in matrix form:

$$
\underline{E}^{s}=\left(\begin{array}{c}
E_{\beta}^{s} \\
E_{\phi}^{s}
\end{array}\right)=\underline{\underline{M}}_{1}\left(\begin{array}{c}
E_{\|}^{i} \\
E_{\perp}^{i}
\end{array}\right) I^{s}=\underline{\underline{M}}_{1} \underline{\underline{M}}_{0}\left(\begin{array}{c}
E_{\beta^{\prime}}^{i} \\
E_{\phi^{\prime}}^{i}
\end{array}\right) I^{s}=\underline{\underline{M}}\left(\begin{array}{c}
E_{\beta^{\prime}}^{i} \\
E_{\phi^{\prime}}^{i}
\end{array}\right) I^{s} .
$$

The matrices $\underline{\underline{M}}_{0}$ and $\underline{\underline{M_{1}}}$ involved in the above expression are reported in the appendix and account for the GO response (i.e., reflection and transmission) of the structure and for changes of local coordinate systems. After analytical integrations and representations, the integral $I^{s}$ can be recast as:

$$
I^{s}=\frac{\mathrm{e}^{-j k_{0} z \cos \beta^{\prime}}}{2 \sin \beta^{\prime}} \frac{1}{2 \pi j} \int_{C} \frac{\mathrm{e}^{-j k_{0} \rho \sin \beta^{\prime} \cos (\alpha \mp \phi)}}{\cos \alpha+\cos \phi^{\prime}} d \alpha,
$$

where $C$ is the integration path in the complex $\alpha$-plane shown in Figure 2. Then, the Steepest Descent Method is applied to $I^{s}$, and a uniform asymptotic evaluation of the integral along the steepest descent path furnishes the diffraction term $I^{d}$. As a consequence, the diffracted field $\underline{E}^{d}$ can be so expressed:

$$
\underline{E}^{d}=\left(\begin{array}{c}
E_{\beta}^{d} \\
E_{\phi}^{d}
\end{array}\right)=\underline{\underline{M}}\left(\begin{array}{c}
E_{\beta^{\prime}}^{i} \\
E_{\phi^{\prime}}^{i}
\end{array}\right) I^{d}=\underline{\underline{D}}\left(\begin{array}{c}
E_{\beta^{\prime}}^{i} \\
E_{\phi^{\prime}}^{i}
\end{array}\right) \frac{e^{-j k_{0} s}}{\sqrt{s}},
$$

where $s$ is the distance along the diffraction direction and the $2 \times 2$ diffraction matrix $\underline{\underline{D}}$ is

$$
\begin{aligned}
\underline{\underline{D}}= & \frac{1}{2 \sqrt{2 \pi k_{0}}} \frac{\mathrm{e}^{-j \pi / 4}}{\sin ^{2} \beta^{\prime}\left(\cos \phi+\cos \phi^{\prime}\right)} \\
& F_{t}\left(2 k_{0} s \sin ^{2} \beta^{\prime} \cos ^{2}\left(\frac{\phi \pm \phi^{\prime}}{2}\right)\right) \underline{\underline{M}}
\end{aligned}
$$

in which $F_{t}$ is the UTD transition function [2] and + (-) sign applies when $0<\phi<\pi(\pi<\phi<2 \pi)$.

A set of representative results is reported. They concern a slab characterised by $\varepsilon_{r}=4-j 0.23$ and $d=0.15 \lambda_{0}\left(\lambda_{0}\right.$ is the free-space wavelength) when a plane wave impinges on it and the observation point moves on a circular path with $\rho=7 \lambda_{0}$. Figure 3 shows the $\beta$-component magnitudes of the GO field and the UAPO diffracted field when $E_{\beta^{\prime}}^{i}=1$, $E_{\phi^{\prime}}^{i}=0$ and $\beta^{\prime}=45^{\circ}, \phi^{\prime}=60^{\circ}$. As expected, the GO pattern presents two discontinuities in correspondence of the incidence and reflection shadow boundaries at $\phi=240^{\circ}$ and $\phi=120^{\circ}$, respectively. The UAPO field contribution is not negligible near such boundaries and guarantees the continuity of the total field across them as shown in Figure 4. The case of normal incidence is considered in the following tests as a useful scenario for comparing the UAPO-based results with those provided by the RF module of COMSOL MULTIPHYSICS [11]. As can be seen in Figures 5 and 6 , an excellent agreement is attained, thus confirming the effectiveness of the UAPO solution.

Accounting for the linearity of the PO radiation integral, the UAPO-based approach for the diffraction problem involving one truncated dielectric slab can be extended to junctions by considering the diffraction contributions of the layers separately. Accordingly, in the case of junctions formed by two slabs, it results:

$$
\begin{aligned}
\underline{E}^{s}= & -j k_{0} \iint_{S_{1}+S_{2}}\left[(\underline{I}-\hat{R} \hat{R}) \zeta_{0} J_{s}^{\mathrm{PO}}+\underline{J}_{m s}^{\mathrm{PO}} \times \hat{R}\right] G\left(\underline{r}, \underline{r}^{\prime}\right) d S \\
= & -j k_{0} \iint_{S_{1}}\left[(\underline{\underline{I}}-\hat{R} \hat{R}) \zeta_{0} J_{s_{1}}^{\mathrm{PO}}+\underline{J}_{m s_{1}}^{\mathrm{PO}} \times \hat{R}\right] G\left(\underline{r}, \underline{r}^{\prime}\right) d S_{1} \\
& -j k_{0} \int_{S_{2}}\left[(\underline{I}-\hat{R} \hat{R}) \zeta_{0} J_{s_{2}}^{\mathrm{PO}}+\underline{J}_{m s_{2}}^{\mathrm{PO}} \times \hat{R}\right] G\left(\underline{r}, \underline{r}^{\prime}\right) d S_{2} \\
= & \underline{E}_{1}^{s}+\underline{E}_{2}^{s}
\end{aligned}
$$




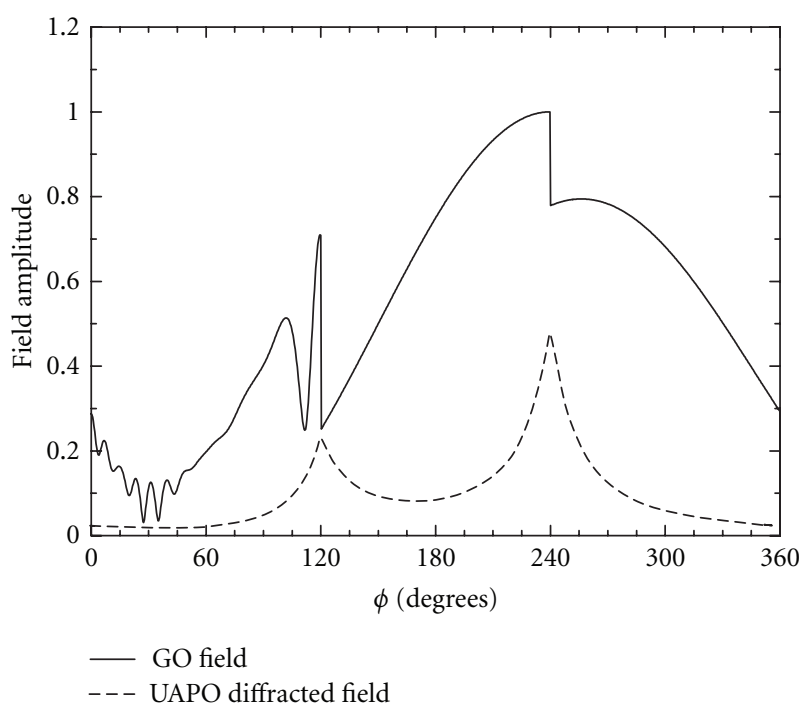

Figure 3: Dielectric slab. The $\beta$-component magnitudes of the GO field and the UAPO diffracted field at $\rho=7 \lambda_{0}$ when $\varepsilon_{r}=4-j 0.23$, $d=0.15 \lambda_{0}, \beta^{\prime}=45^{\circ}, \phi^{\prime}=60^{\circ}$ and $E_{\beta^{\prime}}^{i}=1, E_{\phi^{\prime}}^{i}=0$.

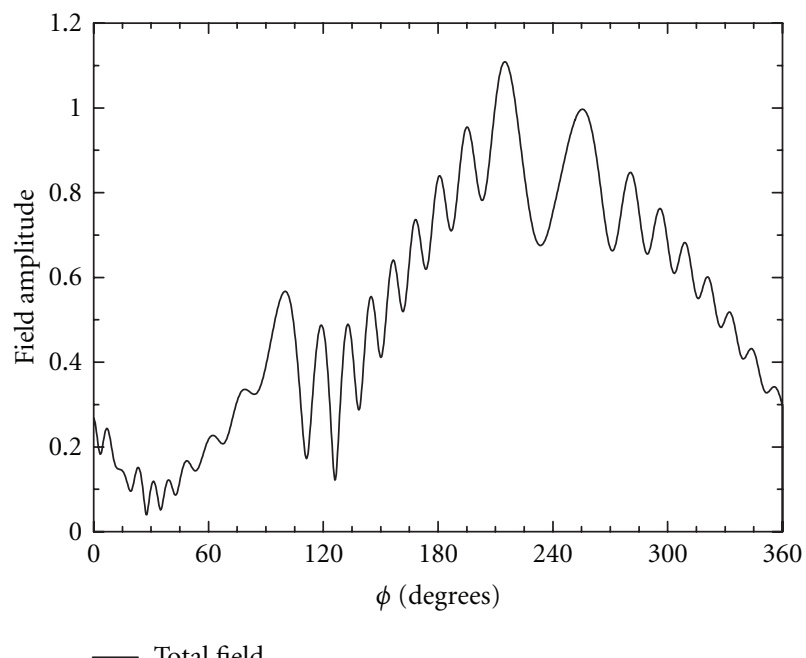

FIGURE 4: Dielectric slab. The $\beta$-component magnitude of the total field at $\rho=7 \lambda_{0}$ when $\varepsilon_{r}=4-j 0.23, d=0.15 \lambda_{0}, \beta^{\prime}=45^{\circ}, \phi^{\prime}=60^{\circ}$ and $E_{\beta^{\prime}}^{i}=1, E_{\phi^{\prime}}^{i}=0$.

and then $\underline{\underline{D}}=\underline{\underline{D}}_{1}+\underline{\underline{D}}_{2}$, with $\underline{\underline{D}}_{1}$ given by (11). The diffraction matrix $\underline{\underline{D}}_{2}$ related to the wave phenomenon originated by the second layer forming the junction can be determined by taking into account that if the external angle of the junction is $n \pi$, a $(n-1) \pi$ rotation of the edge-fixed coordinate system must be considered.

\section{Dielectric Wedges}

A two-dimensional scattering scenario involving a lossless nonmagnetic dielectric wedge with obtuse apex angle $\alpha=$ $(2-n) \pi$ is sketched in Figure 7. Since $\beta^{\prime}=90^{\circ}$, the incident

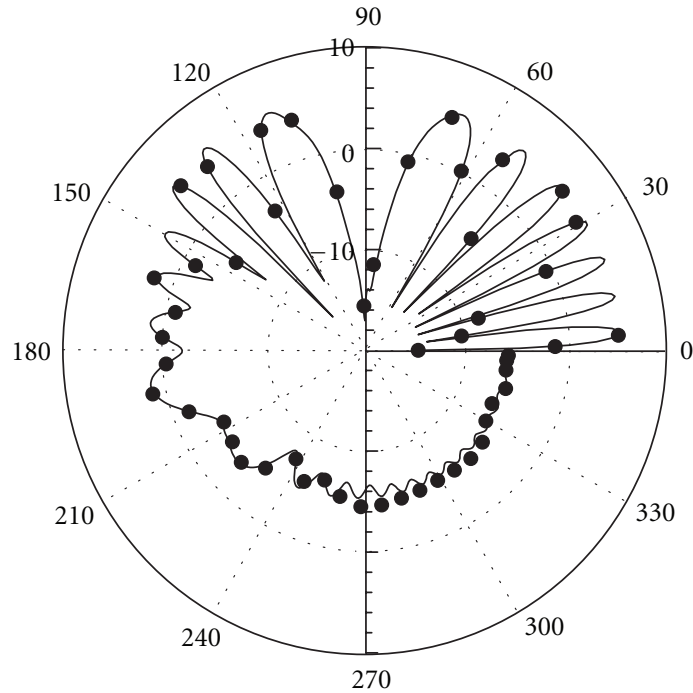

- UAPO-based approach

- Comsol multiphysics

FIgUre 5: Dielectric slab. The relative magnitude of the total field $\beta$-component at $\rho=7 \lambda_{0}$ when $\varepsilon_{r}=4-j 0.23, d=0.15 \lambda_{0}, \beta^{\prime}=90^{\circ}$, $\phi^{\prime}=30^{\circ}$ and $E_{\beta^{\prime}}^{i}=1, E_{\phi^{\prime}}^{i}=0$.

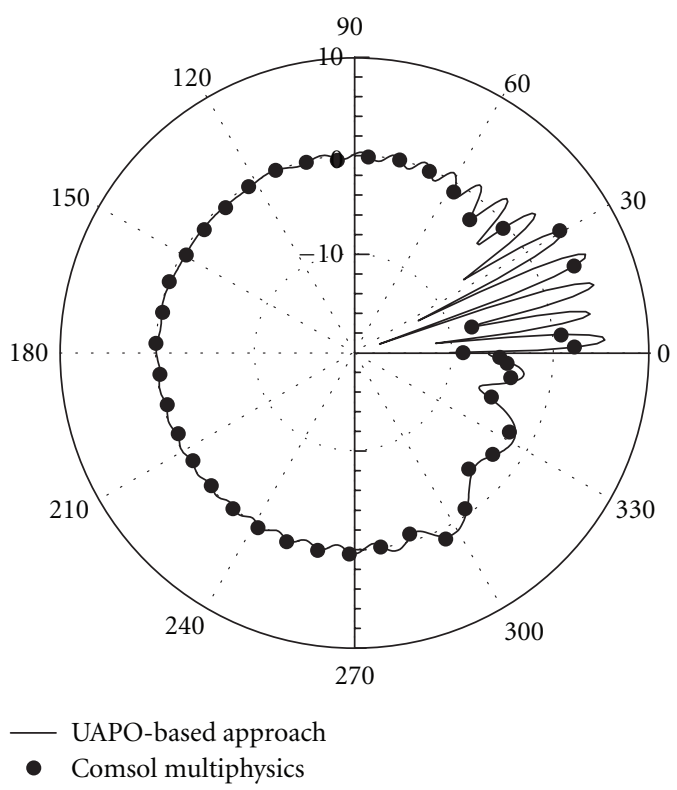

FIGURE 6: Dielectric slab. The relative magnitude of the total field $\beta$-component at $\rho=7 \lambda_{0}$ when $\varepsilon_{r}=4-j 0.23, d=0.15 \lambda_{0}, \beta^{\prime}=90^{\circ}$, $\phi^{\prime}=140^{\circ}$ and $E_{\beta^{\prime}}^{i}=1, E_{\phi^{\prime}}^{i}=0$.

electric field is resolved in two orthogonal components directed along $\hat{z}$ and $\hat{\phi}^{\prime}$.

Note that the two-dimensional diffraction problem involving a right-angled dielectric wedge has been tackled and solved by the authors in [12]. In addition, a UAPO solution for the three-dimensional diffraction problem originated by a nonpenetrable arbitrary-angled wedge with finite conductivity can be found in [13]. 


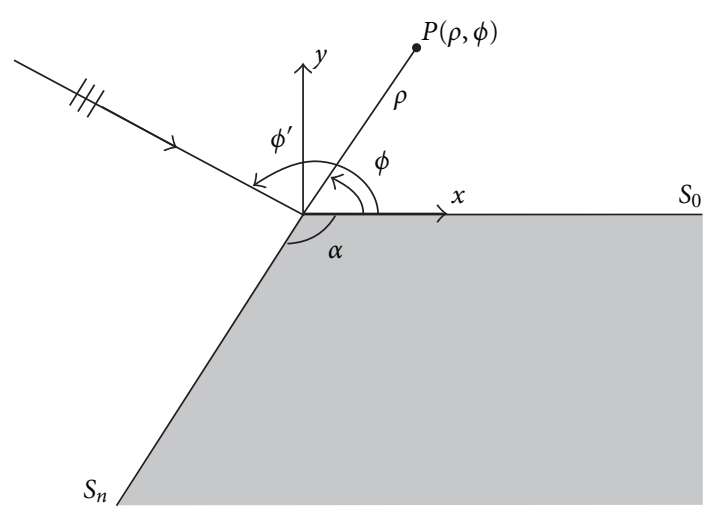

FIGURE 7: Diffraction by an obtuse-angled dielectric wedge.

The methodology adopted for penetrable wedges identifies the inner region (dielectric material) and the surrounding space as separate observation domains and the scattered electric field in each region as originated by electric and magnetic equivalent PO surface currents located on the inner/outer faces of $S_{0}$ and $S_{n}$. According to $[12,14]$, the UAPO diffracted field can be so expressed:

$$
\begin{aligned}
\left(\begin{array}{c}
E_{z}^{d} \\
E_{\phi}^{d}
\end{array}\right) & =\left(\begin{array}{cc}
D_{z z} & 0 \\
0 & D_{\phi \phi^{\prime}}
\end{array}\right)\left(\begin{array}{c}
E_{z}^{i} \\
E_{\phi^{\prime}}^{i}
\end{array}\right) \frac{\mathrm{e}^{-j k \rho}}{\sqrt{\rho}} \\
& =\left(\begin{array}{cc}
D_{z z_{0}}+D_{z z_{n}} & 0 \\
0 & D_{\phi \phi^{\prime}{ }_{0}}+D_{\phi \phi^{\prime}{ }_{n}}
\end{array}\right)\left(\begin{array}{c}
E_{z}^{i} \\
E_{\phi^{\prime}}^{i}
\end{array}\right) \frac{\mathrm{e}^{-j k \rho}}{\sqrt{\rho}} .
\end{aligned}
$$

The analytical expressions of the diffraction matrix elements in (13) depend on the incidence direction and the geometric and electric characteristics of the wedge. In the following, the terms $R_{0, n}$ and $T_{0, n}$ denote the standard reflection and transmission coefficients [10] related to $S_{0}$ and $S_{n}$. The incidence direction defines two cases: (1) both the faces of the wedge are illuminated; (2) only one face is illuminated.

Case $1\left((n-1) \pi<\phi^{\prime}<\pi\right)$. Both the faces of the wedge are illuminated by the impinging wave, and the GO field presents two reflection boundaries in the space surrounding the wedge and two transmission boundaries in the dielectric material.

\section{(i) Outer region:}

$$
\begin{aligned}
D_{z z_{0}}^{\text {out }} & =\left[\left(1-R_{0 \perp}\right) \sin \phi^{\prime}-\left(1+R_{0 \perp}\right) \sin \phi\right] h_{0}^{\text {out }}, \\
D_{\phi \phi^{\prime}}^{\text {out }} & =\left[-\left(1-R_{0 \|}\right) \sin \phi^{\prime}+\left(1+R_{0 \|}\right) \sin \phi\right] h_{0}^{\text {out }}, \\
h_{0}^{\text {out }} & =\frac{\mathrm{e}^{-j \pi / 4}}{2 \sqrt{2 \pi k_{0}}} \frac{F_{t}\left(2 k_{0} \rho \cos ^{2}\left(\left(\phi \pm \phi^{\prime}\right) / 2\right)\right)}{\cos \phi+\cos \phi^{\prime}},
\end{aligned}
$$

where $+(-)$ sign applies when $0<\phi<\pi(\pi<\phi<$ $n \pi)$, and

$$
\begin{aligned}
D_{z z_{n}}^{\text {out }}= & {\left[\left(1-R_{n \perp}\right) \sin \left(n \pi-\phi^{\prime}\right)-\left(1+R_{n \perp}\right) \sin (n \pi-\phi)\right] h_{n}^{\text {out }}, } \\
D_{\phi \phi_{n}^{\prime}}^{\text {out }}= & {\left[-\left(1-R_{n \|}\right) \sin \left(n \pi-\phi^{\prime}\right)+\left(1+R_{n \|}\right) \sin (n \pi-\phi)\right] } \\
& \times h_{n}^{\text {out }}, \\
h_{n}^{\text {out }}= & \frac{\mathrm{e}^{-j \pi / 4}}{2 \sqrt{2 \pi k_{0}}} \\
& \frac{F_{t}\left(2 k_{0} \rho \cos ^{2}\left(\left((n \pi-\phi) \pm\left(n \pi-\phi^{\prime}\right)\right) / 2\right)\right)}{\cos (n \pi-\phi)+\cos \left(n \pi-\phi^{\prime}\right)}
\end{aligned}
$$

in which the sign $+(-)$ holds for $(n-1) \pi<\phi<$ $n \pi(0<\phi<(n-1) \pi)$.

(ii) Inner region:

$$
\begin{aligned}
& D_{z z_{0}}^{\text {in }}=\left[\sqrt{\varepsilon_{r}} \sin \phi-\sqrt{\varepsilon_{r}-\cos ^{2} \phi^{\prime}}\right] T_{0 \perp} h_{0}^{\text {in }}, \\
& D_{\phi \phi_{0}^{\prime}}^{\text {out }}=-\left[\sqrt{\varepsilon_{r}} \sin \phi-\sqrt{\varepsilon_{r}-\cos ^{2} \phi^{\prime}}\right] T_{0 \|} h_{0}^{\text {in }}, \\
& h_{0}^{\text {in }}=\frac{e^{-j \pi / 4}}{2 \sqrt{2 \pi k}} \frac{F_{t}\left(2 k \rho \cos ^{2}\left(\left(\phi-\cos ^{-1}\left(\cos \phi^{\prime} / \sqrt{\varepsilon_{r}}\right)\right) / 2\right)\right)}{\sqrt{\varepsilon_{r}} \cos \phi+\cos \phi^{\prime}}, \\
& D_{z z_{n}}^{\text {in }}=\left[\sqrt{\varepsilon_{r}} \sin (n \pi-\phi)-\sqrt{\varepsilon_{r}-\cos ^{2}\left(n \pi-\phi^{\prime}\right)}\right] T_{n \perp} h_{n}^{\text {in }}, \\
& D_{\phi \phi^{\prime}}^{\text {in }}=-\left[\sqrt{\varepsilon_{r}} \sin (n \pi-\phi)-\sqrt{\varepsilon_{r}-\cos ^{2}\left(n \pi-\phi^{\prime}\right)}\right] T_{n \|} h_{n}^{\text {in }}, \\
& h_{n}^{\text {in }}=\frac{e^{-j \pi / 4}}{2 \sqrt{2 \pi k}} \\
& \frac{F_{t}\left(2 k \rho \cos ^{2}\left(\left(n \pi-\phi-\cos ^{-1}\left(\cos \left(n \pi-\phi^{\prime}\right) / \sqrt{\varepsilon_{r}}\right)\right) / 2\right)\right)}{\sqrt{\varepsilon_{r}} \cos (n \pi-\phi)+\cos \left(n \pi-\phi^{\prime}\right)} .
\end{aligned}
$$

Case $2\left(0<\phi^{\prime}<(n-1) \pi\right)$. Only the external face of $S_{0}$ is illuminated by the incident wave, and two different cases must be separately accounted for.

Subcase $1\left(0<\phi^{\prime}<(n-1) \pi\right.$ and $\left.\cos \phi^{\prime}<\sqrt{\varepsilon_{r}}|\cos (n \pi)|\right)$. The field transmitted through $S_{0}$ does not illuminate the internal face of $S_{n}$ and therefore $D_{z z_{n}}=D_{\phi \phi^{\prime}}=0$. Shadow boundaries related to the incident and reflected fields appear in the external region, and only a transmission shadow boundary is present in the wedge. The expressions of $D_{z z_{0}}$ and $D_{\phi \phi_{0}^{\prime}}$ are equal to those reported for the Case 1 .

Subcase $2\left(0<\phi^{\prime}<(n-1) \pi\right.$ and $\left.\cos \phi^{\prime}>\sqrt{\varepsilon_{r}}|\cos (n \pi)|\right)$. The field transmitted through $S_{0}$ penetrates in the dielectric region and illuminates the internal face of $S_{n}$. It is subsequently transmitted through $S_{n}$ with coefficient $T_{0 n}$ and reflected with coefficient $R_{0 n}$. Accordingly, shadow boundaries related so the incident, reflected, and transmitted-transmitted fields are in the space surrounding the wedge, whereas only the shadow boundary associated to the transmittedreflected field exists in the dielectric material. 


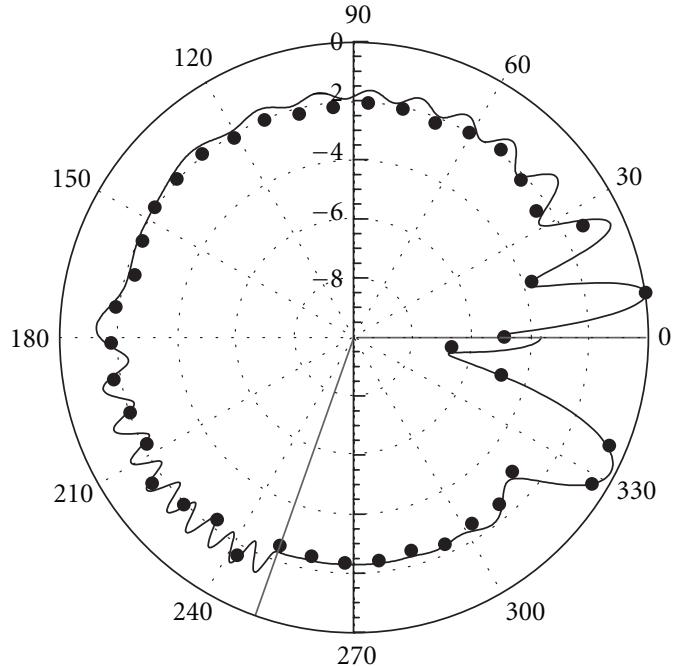

- UAPO-based approach

- FDTD

Figure 8: Dielectric wedge. The relative magnitude of the total field $z$-component at $\rho=5 \lambda_{0}$ when $\alpha=110^{\circ}, \varepsilon_{r}=1.25$, and $\phi^{\prime}=160^{\circ}$.

(i) Outer region:

$$
\begin{aligned}
D_{z z_{n}}^{\text {out }}= & -\left[\cos \theta_{n}^{t}+\sin (n \pi-\phi)\right] T_{0 \perp} T_{0 n_{\perp}} h_{n}^{\text {out }} \\
D_{\phi \phi_{n}^{\prime}}^{\text {out }}= & {\left[\cos \theta_{n}^{t}+\sin (n \pi-\phi)\right] T_{0 \|} T_{0 n \|} h_{n}^{\text {out }} } \\
h_{n}^{\text {out }}= & \frac{\mathrm{e}^{-j \pi / 4}}{2 \sqrt{2 \pi k_{0}}} \\
& \frac{F_{t}\left(2 k_{0} \rho \cos ^{2}\left(\left(\cos ^{-1}(-\eta) \pm(\phi+(1-n) \pi)\right) / 2\right)\right)}{\cos (n \pi-\phi)+\eta},
\end{aligned}
$$

where

$$
\begin{aligned}
& \theta_{n}^{t}=\sin ^{-1}\left(\sqrt{\varepsilon_{r}} \sin \left((2-n) \pi-\sin ^{-1}\left(\cos \phi^{\prime} / \sqrt{\varepsilon_{r}}\right)\right)\right), \\
& \eta=\cos (n \pi) \cos \phi^{\prime}+\sin (n \pi) \sqrt{\varepsilon_{r}-\cos ^{2} \phi^{\prime}}
\end{aligned}
$$

and the $+(-)$ sign holds when $(n-1) \pi<\phi<n \pi(0<\phi<$ $(n-1) \pi)$. The expressions of $D_{z z_{0}}^{\text {out }}$ and $D_{\phi \phi^{\prime}}^{\text {out }}$ are equal to those presented for the Case 1 . Note that the total reflection occurs on $S_{n}$ when $\cos \phi^{\prime} \leq\left|\cos (n \pi)+\sin (n \pi) \sqrt{\varepsilon_{r}-1}\right|$. In such a case it is appropriate to set $D_{z z_{n}}^{\text {out }}=D_{\phi \phi_{n}^{\prime}}^{\text {out }}=0$, since the surface waves are not taken into account in the UAPO-based approach.

(ii) Inner region:

$$
\begin{aligned}
D_{z z_{n}}^{\text {in }}= & {\left[\left(1-R_{0 n_{\perp}}\right) \cos \left(n \pi+\theta_{0}^{t}\right)+\left(1+R_{0 n_{\perp}}\right) \sin (n \pi-\phi)\right] } \\
\times & \sqrt{\varepsilon_{r}} T_{0 \perp} h_{n}^{\text {in }}, \\
D_{\phi \phi^{\prime}{ }_{n}}^{\text {in }}=- & {\left[\left(1-R_{0 n \|}\right) \cos \left(n \pi+\theta_{0}^{t}\right)+\left(1+R_{0 n \|}\right)\right.} \\
& \sin (n \pi-\phi)] \sqrt{\varepsilon_{r}} T_{0 \|} h_{n}^{\text {in }},
\end{aligned}
$$

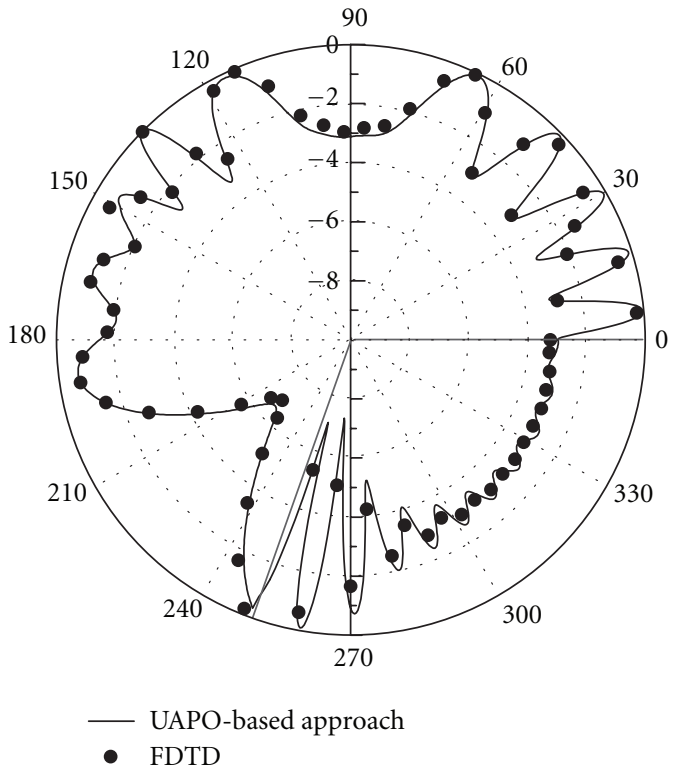

FIGURE 9: Dielectric wedge. The relative magnitude of the total field $z$-component at $\rho=5 \lambda_{0}$ when $\alpha=110^{\circ}, \varepsilon_{r}=1.25$, and $\phi^{\prime}=30^{\circ}$.

$$
\begin{aligned}
h_{n}^{\text {in }} & =\frac{\mathrm{e}^{-j \pi / 4}}{2 \sqrt{2 \pi k}} \\
& \frac{F_{t}\left(2 k \rho \cos ^{2}\left(\left(\cos ^{-1}\left(-\eta / \sqrt{\varepsilon_{r}}\right)-(\phi+(1-n) \pi)\right) / 2\right)\right)}{\sqrt{\varepsilon_{r}} \cos (n \pi-\phi)+\eta},
\end{aligned}
$$

in which $\theta_{0}^{t}=\sin ^{-1}\left(\cos \phi^{\prime} / \sqrt{\varepsilon_{r}}\right)$. The expressions of $D_{z z_{0}}^{\text {in }}$ and $D_{\phi \phi^{\prime}{ }_{0}}^{\text {in }}$ are equal to those presented for the Case 1.

Figures 8 and 9 show comparisons with data provided by an "ad hoc" developed code implementing the FiniteDifference Time-Domain (FDTD) method [15]. They refer to a wedge characterised by $\alpha=110^{\circ}$ and $\varepsilon_{r}=1.25$ when the observation point moves along a circular path with $\rho=5 \lambda_{0}$. The good agreements assess the effectiveness of the presented UAPO solution also when considering dielectric wedges.

Note that the case corresponding to $\pi<\phi^{\prime}<n \pi$ is not reported here because it can be tackled and solved in a similar way.

\section{Conclusions}

UAPO solutions have been presented in the UTD context for evaluating the field diffracted by penetrable dielectric slabs and wedges. They are in closed form, simple and easy to handle and yield total field levels in good agreement with data obtained via numerical tools. In addition, the UTD-like formulation of UAPO diffraction coefficients facilitates the analytical evaluation of the time domain counterparts. These characteristics encourage the use of UAPO solutions for diffraction problems of interest in many application areas. Future research activities will be directed towards threedimensional scenarios involving penetrable wedges with finite conductivity. 


\section{Appendix}

$$
\begin{aligned}
& \underline{\underline{M}}_{0}=\frac{1}{\sqrt{1-\sin ^{2} \beta^{\prime} \sin ^{2} \phi^{\prime}}}\left(\begin{array}{cc}
\cos \beta^{\prime} \sin \phi^{\prime} & \cos \phi^{\prime} \\
-\cos \phi^{\prime} & \cos \beta^{\prime} \sin \phi^{\prime}
\end{array}\right), \\
& \underline{\underline{M}}_{1}=\underline{\underline{M}}_{7}\left[\underline{\underline{M}}_{2} \underline{\underline{M}}_{4} \underline{\underline{M}}_{5}+\underline{\underline{M}}_{3} \underline{\underline{M}}_{4} \underline{\underline{M}}_{6}\right] \text {, } \\
& \underline{\underline{M}}_{7}=\left(\begin{array}{ccc}
\cos \beta^{\prime} \cos \phi & \cos \beta^{\prime} \sin \phi & -\sin \beta^{\prime} \\
-\sin \phi & \cos \phi & 0
\end{array}\right) \text {, } \\
& \underline{\underline{M}}_{2}=\left(\begin{array}{cc}
1-\sin ^{2} \beta^{\prime} \cos ^{2} \phi & -\sin \beta^{\prime} \cos \beta^{\prime} \cos \phi \\
-\sin ^{2} \beta^{\prime} \sin \phi \cos \phi & -\sin \beta^{\prime} \cos \beta^{\prime} \sin \phi \\
-\sin \beta^{\prime} \cos \beta^{\prime} \cos \phi & \sin ^{2} \beta^{\prime}
\end{array}\right) \text {, } \\
& \underline{\underline{M}}_{3}=\left(\begin{array}{cc}
0 & -\sin \beta^{\prime} \sin \phi \\
-\cos \beta^{\prime} & \sin \beta^{\prime} \cos \phi \\
\sin \beta^{\prime} \sin \phi & 0
\end{array}\right) \text {, } \\
& \underline{\underline{M}}_{4}=\frac{1}{\sqrt{1-\sin ^{2} \beta^{\prime} \sin ^{2} \phi^{\prime}}}\left(\begin{array}{cc}
-\cos \beta^{\prime} & -\sin \beta^{\prime} \cos \phi^{\prime} \\
-\sin \beta^{\prime} \cos \phi^{\prime} & \cos \beta^{\prime}
\end{array}\right) \text {, } \\
& \underline{\underline{M}}_{5}=\left(\begin{array}{cc}
0 & \left(1-R_{\perp}-T_{\perp}\right) \cos \theta^{i} \\
1+R_{\|}-T_{\|} & 0
\end{array}\right) \text {, } \\
& \underline{\underline{M}}_{6}=\left(\begin{array}{cc}
\left(1-R_{\|}-T_{\|}\right) \cos \theta^{i} & 0 \\
0 & -\left(1+R_{\perp}-T_{\perp}\right)
\end{array}\right) \text {. }
\end{aligned}
$$

[10] C. A. Balanis, Advanced Engineering Electromagnetics, Wiley, New York, NY, USA, 1989.

[11] COMSOL Multiphysics, v. 3.5a, Users' guide, RF Module, 2008.

[12] G. Gennarelli and G. Riccio, "A uniform asymptotic solution for the diffraction by a right-angled dielectric wedge," IEEE Transactions on Antennas and Propagation, vol. 59, no. 3, Article ID 5677594, pp. 898-903, 2011.

[13] G. Gennarelli and G. Riccio, "A UAPO-based model for propagation prediction in microcellular environments," Progress In Electromagnetics Research B, no. 17, pp. 101-116, 2009.

[14] G. Gennarelli and G. Riccio, "Plane-wave diffraction by an obtuse-angled dielectric wedge," Journal of the Optical Society of America A, vol. 28, no. 4, pp. 627-632, 2011.

[15] A. Taflove and S. Hagness, Computational Electrodynamics: The Finite Difference Time Domain Method, Artech House, London, UK, 2000.

\section{References}

[1] J. B. Keller, "Geometrical theory of diffraction," Journal of the Optical Society of America, vol. 52, pp. 116-130, 1962.

[2] R. G. Kouyoumjian and P. H. Pathak, "A uniform geometrical theory of diffraction for an edge in a perfectly conducting surface," Proceedings of the IEEE, vol. 62, no. 11, pp. 14481461, 1974.

[3] P. C. Chang, R. J. Burkholder, J. L. Volakis, R. J. Marhefka, and Y. Bayram, "High-frequency em characterization of throughwall building imaging," IEEE Transactions on Geoscience and Remote Sensing, vol. 47, no. 5, Article ID 4810144, pp. 13751387, 2009.

[4] A. I. Kozlov, L. Lighart, and A. I. Logvin, "Radar reflection from surfaces with ruptures," in Proceedings of 13th International Conference on Microwaves, Radar and Wireless Communications (MIKON '00), vol. 1, pp. 347-350, 2000.

[5] W. D. Burnside and K. W. Burgener, "High frequency scattering by a thin lossless dielectric slab," IEEE Transactions on Antennas and Propagation, vol. 31, no. 1, pp. 104-110, 1983.

[6] R. J. Leubbers, "Finite conductivity uniform GTD versus knife edge diffraction in prediction of propagation path loss," IEEE Transactions on Antennas and Propagation, vol. 32, no. 1, pp. 70-76, 1984.

[7] P. Bernardi, R. Cicchetti, and O. Testa, "A three-dimensional UTD heuristic diffraction coefficient for complex penetrable wedges," IEEE Transactions on Antennas and Propagation, vol. 50, no. 2, pp. 217-224, 2002.

[8] T. W. Veruttipong, "Time domain version of the uniform GTD," IEEE Transactions on Antennas and Propagation, vol. 38, no. 11, pp. 1757-1764, 1990.

[9] F. Ferrara, C. Gennarelli, G. Gennarelli, M. Migliozzi, and G. Riccio, "Scattering by truncated lossy layers: A UAPO-based approach," Electromagnetics, vol. 27, no. 7, pp. 443-456, 2007. 

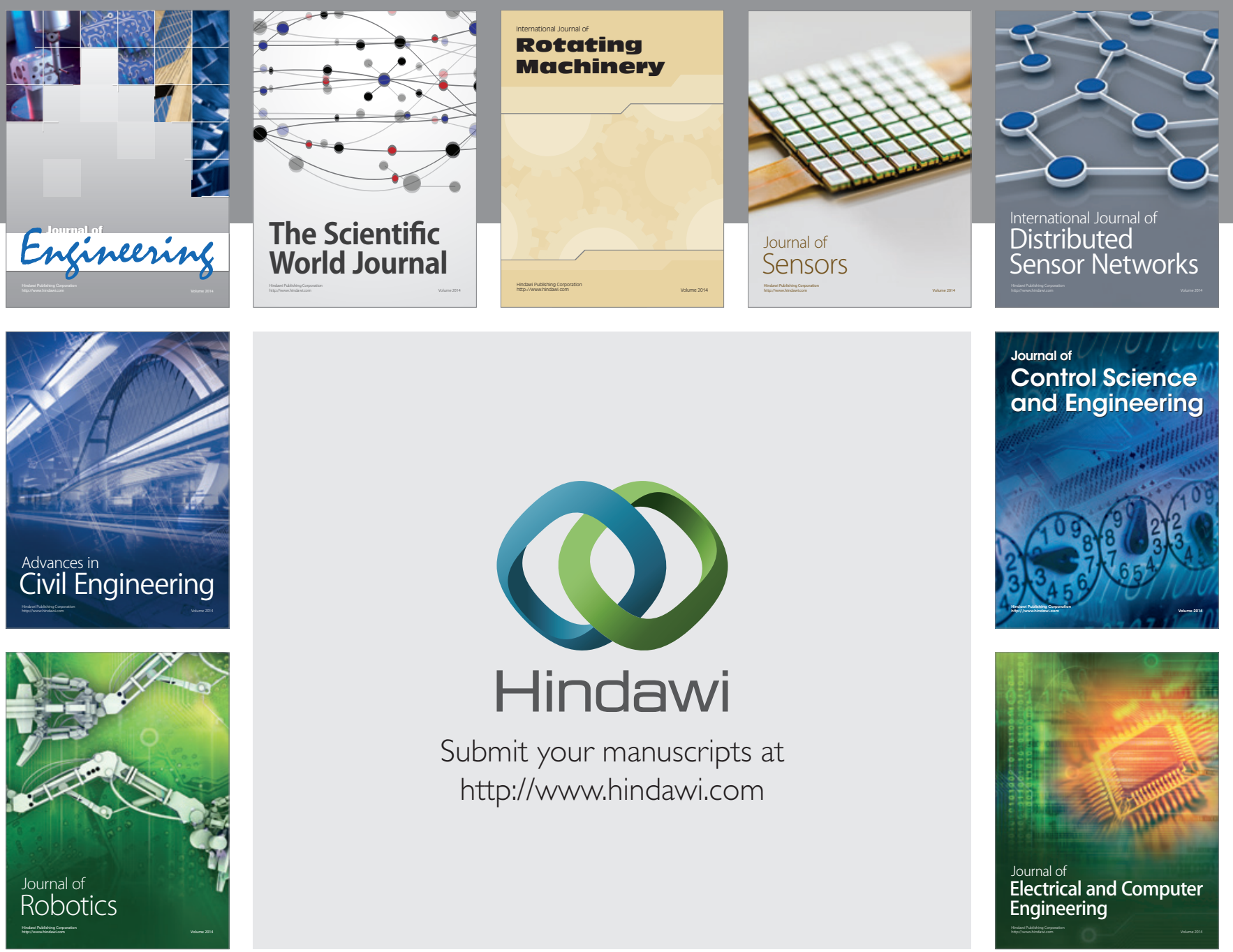

Submit your manuscripts at

http://www.hindawi.com
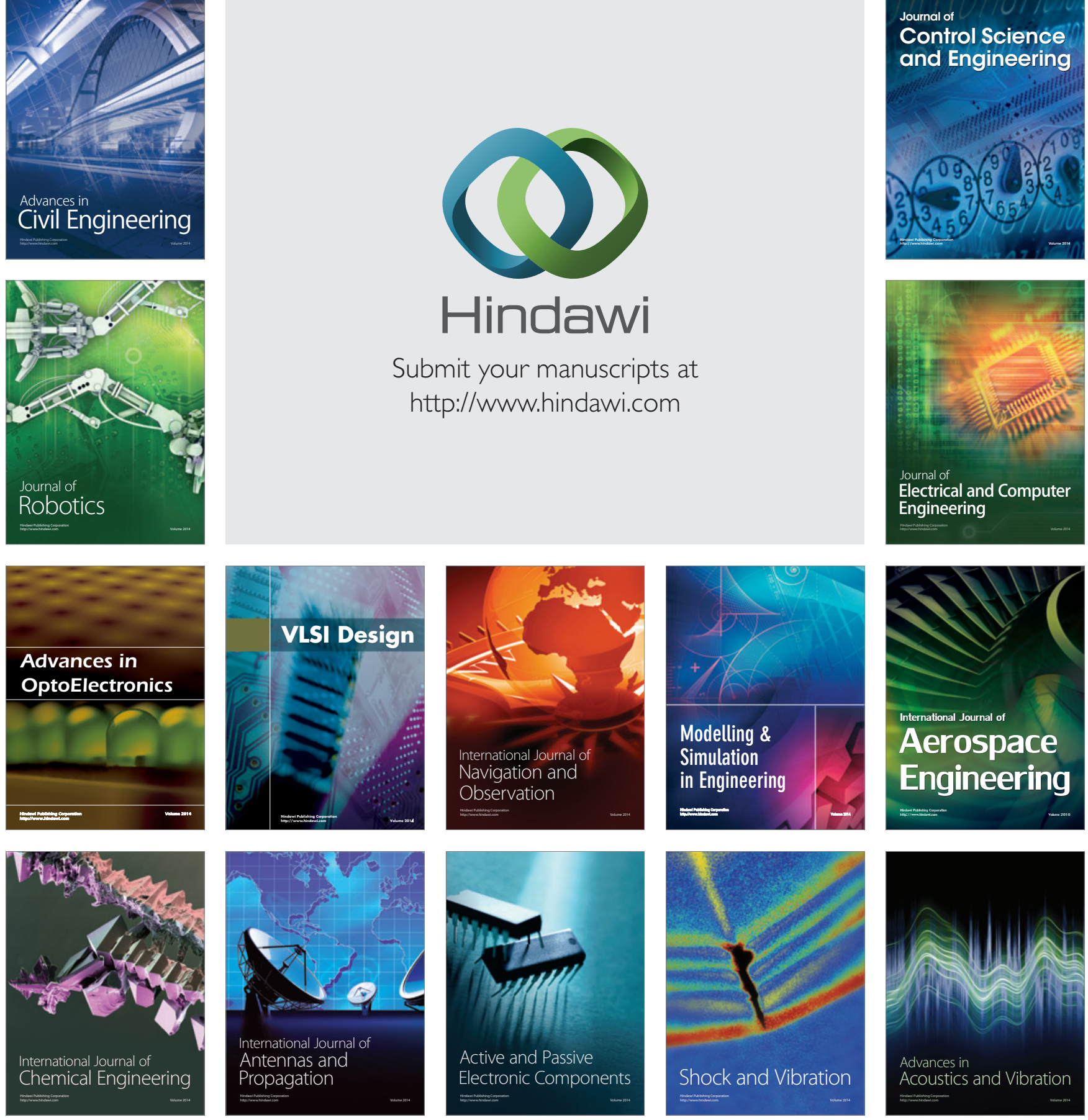